\title{
La Educación. Un factor determinante de la estabilización del desarrollo local sostenible
}

\author{
Venegas, Gina* \\ La Calle, Juan José* \\ Proaño, Carlos** \\ *Universidad Técnica de Cotopaxi, Latacunga, Ecuador \\ **Universidad de las Fuerzas Armadas ESPE, Latacunga, Ecuador \\ gina.venegas@utc.edu.ec
}

Recibido: 18 abril 2018 Aprobado: 22 mayo 2018

\begin{abstract}
Resumen
La educación es un instrumento esencial en el desarrollo local, pero no puede convertirse en el único elemento responsable del mismo. Actualmente se habla de la influencia de las competencias educacionales como aliciente de un mejoramiento del plano económico social. Se empieza a endosar la responsabilidad de un futuro éxito económico-social-político de la región y del país al proceso educativo enmarcado en un modelo pedagógico que facilite la solución de problemáticas socioeconómicas. Un primer problema que se plantea ante la relación entre desarrollo local y educación, es ¿Cuál es la pedagogía más adecuada a implementar para lograr un desarrollo local? El objetivo de este trabajo fue identificar la pedagogía adecuada que permita despegar con el desarrollo local. Hoy en día hay una verdadera selva pedagógica difícil de aplicar de manera clara en los procesos educativos; desde las instancias gubernamentales las políticas emanadas se proyectan a buscar un mejoramiento de la calidad educativa con proyección a contribuir con el desarrollo sostenible del país, pero las diferentes escuelas pedagógicas son difíciles de adaptar. Se aplicó una encuesta para determinar la pedagogía más utilizada por los docentes en el aula y como modelo pedagógico institucional y se realizó un análisis documental. El resultado muestra la preferencia docente para desarrollar su actividad académica y se observa claramente que la educación es solo un medio y que necesita adoptar una corriente pedagógica que vaya de la mano con el desarrollo o el postdesarrollo local. Se propone un modelo aplicado a establecimientos educativos franceses.
\end{abstract}

Palabras clave: Educación, Pedagogía, Desarrollo local, Sostenibilidad.

REVISTA DE INVESTIGACIÓN SIGMA / Vol. 05, Nº 1, 2018 (pág. 87-104) 


\title{
Education. A determining factor in the stabilization of sustainable local development
}

\begin{abstract}
Education is an essential instrument in local development, but it can not become the only element responsible for it. At present, the influence of educational competences is discussed as an incentive for an improvement of the social economic level. It is thought that the responsibility of an imminent economic-social-political success of the region and the country depends on the educational process by framed in a pedagogical model that facilitates the solution of socioeconomic problems. A first problem that arises in the relationship between local development and education is: What is the most appropriate pedagogy to be implemented to achieve local development? The objective of this work was to identify the appropriate pedagogy that allows increasing the local development. Nowadays there is a real pedagogical jungle difficult to apply in an appropriate way in the educational processes; from the government offices, the established policies are projected to reach an improvement of the educational quality with projection to contribute with the sustainable development of the country, but the different pedagogical models are difficult to adapt. A survey was applied to determine the pedagogy most used by teachers in the classroom and as an institutional pedagogical model and a documentary analysis was made. The result shows the teachers preference to develop their academic activity and it is clearly observed that education is only an instrument that needs to adopt a pedagogical model compatible with the local development or local postdevelopment. A model applied to French educational establishments is proposed.
\end{abstract}

Keywords: Education, Pedagogy, Local Development, Sustainability.

\section{Introducción}

En el Ecuador, en la actualidad existe un importante debate sobre el papel que el desarrollo local debe tener en el adelanto y progreso de los pueblos. La aparición de estudios de posgrado en diversas universidades, caso de la Universidad Técnica de Cotopaxi, es una muestra de este escenario. Ello se da dentro de una situación muy problemática con cambios importantes en el nivel académico y reestructuración del sistema educativo en general.

Al mismo tiempo se produce un proceso generado por la necesidad de dar respuesta, no solo a la formación de los estudiantes, sino que, por medio de mecanismos sociales, se va sistemáticamente transfiriendo la responsabilidad de contar con un buen orden y funcionamiento de la sociedad en su conjunto, a la educación. Hay una clara transferencia de las responsabilidades sociales desde el plano económico y social al plano educacional social.

En último término se está empezando a pensar que la educación es LA máxima responsable del futuro éxito económico-socialpolítico de los estudiantes de la región y del país. De alguna manera se está planteando que los 
problemas sociales deben ser solucionados desde la educación, no desde la economía.

Joseph Stiglitz y Bruce Greenwald, economistas de profesión, en su obra titulada La Creación de una Sociedad del Aprendizaje manifiestan "El mensaje central es que los gobiernos tienen un papel importante que desempeñar a la hora de moldear una economía innovadora y promover el aprendizaje". (Stiglitz \& Grenwald, 2016)

Así el problema del desarrollo local se está enfocando de manera que los establecimientos escolares deberían asumir la responsabilidad, no de colaborar en dicho desarrollo local, sino lo que es mucho más problemático, ser protagonistas de este desarrollo local.

Aunque neguemos esta responsabilidad desde los establecimientos escolares, hay toda una presión social que se está implementando; ante esta presión es necesario clarificar algunos puntos cruciales.

De hecho, el espacio local se ha convertido en un lugar de visibilización de las llamadas pedagogías sociales, las cuales son a menudo invocadas para que los establecimientos asuman esta nueva responsabilidad frente a las sociedades locales.

\section{¿Cuáles pedagogías?}

El trabajo docente se relaciona íntimamente con la pedagogía. Su aplicación se basa en la concepción de ella y fundamentalmente en el modelo a ejecutarse. Algunos autores definen a Pedagogía como ciencia, arte, saber o disciplina, pero todos están de acuerdo en que se encarga de la educación, es decir, tiene por objeto el planteo, estudio y solución del problema educativo; o también puede decirse que la pedagogía es un conjunto de normas, leyes o principios que se encargan de regular el proceso educativo (Picardo, 2005).

El primer problema con el que se encuentra todo aquel que quiere orientar sus trabajos en establecimientos escolares, es evidentemente el ver qué orientación debe dar a sus clases para que, además de lo habitual, dirigirlas hacia el apoyo del desarrollo local, teniendo siempre como norte el hecho de que debe tratarse de una orientación, más no de una transformación de la educación para convertirla en un apéndice del desarrollo local.

Aquí surge un primer elemento, todos aquellos que están algo familiarizados con la pedagogía se encuentran con lo que cabría definir como la selva pedagógica. ¿Cuáles pedagogías 
son las más adecuadas para colaborar en el desarrollo local?

El recientísimo libro del filósofo y pedagogo José Antonio Marina, titulado "El Bosque Pedagógico" (Marina, 2017) sirve aquí para ejemplificarclaramente esta problemática,así en su página IX, indica una primera clasificación de escuelas, teorías, o sectas pedagógicas, según una clasificación tomada del también estudioso del tema Jaume Trillas: (Trillas, 2007)

“-Pedagogía Progresista de John Dewey.

-Escuela Moderna Ferrer y Guardia.

-Pedagogía Científica de María Montessori.

-Métodos Globales y los Centros de Interés de Ovide Decroly.

-Pedagogía Comunista de Makarenko.

-Pedagogía Antiautoritaria de Neill.

-Propuestas de Psicología Evolutiva de Jean Piaget.

-Propuestas de la Psicología Cultural de Vygotski.

-Tecnología Educativa y métodos conductistas de Skinner.

-Modelo Popular y Cooperativo de Freinet.

-Pedagogía Crítica y las propuestas de desescolarización de Iván Illich.

-Perspectiva de la Sociología de la Educación de Bernstein.
-La educación liberadora de Paulo Freire.” (Díez, 2009)

Para en la página $\mathrm{X}$ indicar una nueva clasificación siguiendo las apreciaciones del pedagogo Rodríguez Neira: (Rodríguez-Neira, 1999)

“-Pedagogía Perenne.

-Pedagogía Performativa.

-Pedagogía Hermenéutica.

-Pedagogía Postestructuralista.

-Pedagogía Psicológica.

-Pedagogía de la Investigación-acción.

-Pedagogía Crítica” (Soler, 2009)

En Pedagogías del Siglo XXI. Alternativas

Para La Innovación Educativa (Carbonell, 2016) el autor clasifica a la pedagogía así:

1. Pedagogías no institucionales.

2. Pedagogías Críticas.

3. Pedagogías Libres no directivas.

4. Pedagogías de la Inclusión y la cooperación.

5. Pedagogías Lentas.

6. Pedagogía Sistémica.

7. Pedagogías del Conocimiento Integrado.

8. Pedagogías de las Diversas Inteligencias.

Como claramente indica Marina, se está 
en una situación problemática. En función de qué criterios, se podría decir que escasamente científicos, se puede decidir qué pedagogía se utiliza o es más apropiada para avanzar en el desarrollo local.

Obviamente hay que dejar a la libertad de cátedra la decisión del profesor, pero evidentemente, según el tipo de establecimiento en el que se labore es muy probable que exista un modelo pedagógico, ya definido y que se tenga que adoptar de manera obligatoria.

La pregunta que surge es la que indica que tal vez ese modelo pedagógico, expresado por el establecimiento, puede o no, estar en concordancia con facilitar el desarrollo local.

Muchos indicios presagian una revolución educativa y requiere de la atención de todas las personas responsables y de todas las instituciones. "El aprendizaje nunca ha sido tan importante como ahora" (Stiglitz \& Grenwald, 2016). Lo que llama la atención sobre este criterio es que Joseph Stiglitz no es un pedagogo, sino un premio Nobel de Economía. La nueva frontera educativa amplía sus límites, coloniza nuevos territorios. (Marina, 2017)

Oscar Picardo Joao, en el Diccionario Pedagógico, dice que "Otros autores como
Ortega y Gasset, ven la pedagogía como una corriente filosófica que llega a ser la aplicación de los problemas referidos a la educación, de una manera de sentir y pensar sobre el mundo" (Picardo, 2005).

\section{Identificando las pedagogías}

Aprovechando la asistencia de profesionales de la enseñanza al primer congreso internacional de ciencias humanas y educación realizado en enero del 2018 en la ciudad de Pujilí, se aplicó una encuesta para conocer el criterio de los participantes en cuanto al desarrollo local sostenible a través de la educación. La población encuestada estuvo conformada por docentes universitarios de varias universidades que asistieron y participaron en el evento académico señalado. La experiencia y formación docente de este grupo de profesionales abaliza, de alguna forma, los resultados que se desprenden de la encuesta.

Se utilizó la encuesta como instrumento de recolección de datos porque permitía analizar las clases de pedagogía desde su concepto hacia su aplicación en el aula y su posterior resultado dentro de la comunidad educativa proyectada hacia la sociedad.

Se decidió utilizar la clasificación citada 
por Marina, como de Rodríguez Neira, pero para evitar confusionismos respecto a qué representan cada escuela, se complementó el nombre con las características suministradas en el artículo Teorías de la educación, De la escuela a la sociedad red: hacia una pedagogía 3.0 de Prats Gil, Enric. Así se transcribió textualmente de dicho artículo las características de cada escuela.
En la encuesta se preguntó específicamente: De las siguientes pedagogías ¿cuál emplea usted habitualmente en el aula, y cual considera usted es un medio para fomentar el desarrollo local sostenible? Se recogieron posteriormente a la conferencia, un total de 115 encuestas con los siguientes resultados, pormenorizados en la tabla 1.

\section{Tabla 1}

Pedagogía utilizada en clase.

\begin{tabular}{lcc}
\multicolumn{1}{c}{ Pedagogías } & Número & Porcentaje \\
\hline Pedagogía Perenne & 5 & 4,35 \\
\hline Pedagogía Performativa & 7 & 6,09 \\
\hline Pedagogía Hermenéutica & 13 & 11,30 \\
\hline Pedagogía Postestructuralista & 0 & 0,00 \\
\hline Pedagogía Psicológica & 12 & 10,43 \\
\hline Pedagogía de la Investigación-Acción & 46 & 40,00 \\
\hline Pedagogía Crítica & 25 & 21,74 \\
\hline Sin respuesta $\quad$ Total & 7 & 6,09 \\
\hline
\end{tabular}

Fuente: Asistentes a Primer Congreso Internacional de Ciencias Humanas y Educación, 2018

Los resultados se reflejan en su análisis de la siguiente manera:

\section{Pedagogía perenne. Definición: "Hay} una manera de hacer pedagogía, y de pensar la educación, basada en la remembranza, en el retorno a unos valores (normalmente, considerados ya perdidos), que hace falta recuperar y volver a instalar en nuestra vida cotidiana. Esta recuperación implica, según sus promotores, situar de nuevo a la persona como centro de las preocupaciones de la pedagogía”. (Prats Gil, Enric, 2010)

De acuerdo a la tabla de porcentualizaciones con respecto a la pedagogía que se utiliza en clase cinco casos que representa el 4,35\% de toda la población encuestada indican que utilizan la pedagogía perenne. Por lo que la gran mayoría no considera a esta pedagogía 
como una opción que contribuya a los docentes en el desarrollo educativo y en el desarrollo local.

\section{Pedagogía performativa. Definición: “Cuando}

la educación se orienta decididamente hacia los productos o resultados de los educandos, nos encontramos ante las pedagogías performativas. Estas pedagogías se sitúan exactamente en el polo opuesto de las pedagogías perennes, puesto que enfatizan precisamente los actos o acciones (performances) del sujeto por encima del individuo mismo, o en el mejor de los casos, a pesar de la persona”. (Prats Gil, Enric, 2010)

De la población encuestada, y; de acuerdo a la tabla de porcentualizaciones, se aprecia que siete casos que representan el $6,09 \%$ ven a la pedagogía performativa como la solución a los problemas educativos. Sin embargo, esta apreciación señala de acuerdo a los resultados que este tipo de pedagogía no es el camino que lleve a un desarrollo local sostenible.

3. Pedagogía hermenéutica. Definición: "La educación es un fenómeno que se explica por la posición que educador y educando tienen en un contexto social, cultural, lingüístico y político limitado históricamente. Bajo una perspectiva hermenéutica, toma fuerza la idea de "el otro", tanto desde la perspectiva del educador (que tiene al educando en frente, como a "otro"), como desde la perspectiva del educando (que también tiene a un educador en frente, pero seguramente tiene más educandos cerca, otros “otros")". (Prats Gil, Enric, 2010

De acuerdo al gráfico y a la tabla de resultados se observa que trece casos que representan el 11,30\% de la población encuestada señala que la pedagogía hermenéutica sí apoyaría en el desarrollo académico y apoyaría al desarrollo local, no obstante, el número en la gráfica general no alcanza para ser la pedagogía a ser usada como recurso imperioso, a pesar de estar relacionada con la parte social.

\section{Pedagogía postestructuralista. Definición:} "El estructuralismo tuvo, a partir de la segunda mitad del siglo XX, un predicamento bastante importante en casi todas las ciencias sociales, especialmente en la lingüística y en la antropología. En educación, sin embargo, la fuerza de las corrientes performativas, precisamente en la misma época, dejó en un segundo plano los argumentos estructuralistas, aunque sí han dejado una cierta huella en el pensamiento pedagógico”. (Prats Gil, Enric, 2010)

De acuerdo a los resultados obtenidos ninguno de los participantes seleccionó a la 
pedagogía posestructuralista como parte de la solución hacia la búsqueda de un desarrollo local sostenible, por esa razón la muestra es de cero, quizá por ser una pedagogía relegada al pasado.

\section{Pedagogía psicológica. Definición: "Como} psicólogo, para mí es muy evidente que, por muy capaces que puedan ser mis colegas, su función no consiste en decidir los objetivos de la educación, como tampoco es la función de los generales decidir si su nación tiene que ir o no a la guerra. [...] Lo que no está tan claro es la distinción entre finalidades y medios, entre los objetivos y los medios para alcanzarlos. Y quizás esto está bien porque los generales tienen un conocimiento intuitivo de lo que es o no posible en la guerra y de contener la amenaza, igual que los psicólogos tienen un cierto conocimiento de cómo podemos hacer que otro aprenda, que preste atención o esté libre de ansiedad. [...] Si el psicólogo no es capaz de hacer su papel de adivinador y diseñador de lo que es posible, no servirá acertadamente a su sociedad". (Prats, 2015)

De acuerdo a las muestras visualizadas en la tabla y el gráfico se advierte que doce casos, que representan el 10,43\% de la población, mira a la pedagogía psicológica como preponderante para alcanzar los objetivos de la educación, sin embargo, a nivel general pierde peso ante la posibilidad de escoger la mejor opción de acuerdo a las pedagogías en el proceso educativo.

\section{Pedagogía de la investigación-acción.}

Definición: "Más que una pedagogía en sentido estricto, la investigación-acción es una metodología de trabajo y una actitud que alerta sobre el carácter investigador del educador. Surgida al amparo de las pedagogías psicológicas y performativas, y con una orientación que la acerca a los enfoques críticos, el concepto de investigación-acción hace referencia a una “[...] forma de indagación autorreflexiva que emprenden los participantes en situaciones sociales para mejorar la racionalidad y la justificación teórica de sus prácticas". (Prats, 2015)

La pedagogía de la investigación- acción ha sido considerada como exitosa para el proceso de cambio y transformación de la educación por ello en referencia a la tabla y al gráfico de porcentualizaciones se refleja que cuarenta y seis encuestados que alcanza el $40 \%$ indican que la pedagogía de la investigación - acción es la más confiable, la más conveniente para mejorar los procesos educativos y el desarrollo local.

7. Pedagogía Crítica. Definición: "Las 
corrientes marxistas, que enfatizaron el papel de la lucha de clases en la configuración de nuestras sociedades y que quedaron relegadas al debate sociocultural tras el mes de mayo del 68, recuperaron aliento con la teoría crítica. De hecho, precisamente Mayo del 68 es el reflejo de un combate entre las posiciones conservadoras inmovilistas, de mantenimiento de la orden, y de los planteamientos anarquizantes que cuestionan las estructuras estatales y dejan el marxismo al margen, sin respuesta, tanto en el debate ideológico, como en el debate estrictamente socioeducativo". (Prats Gil, Enric, 2010)

Una de las pedagogías que alcanza un porcentaje considerable es la pedagogía crítica, se aprecia que 25 encuestados que representan el $21,74 \%$ de la población encuestada, señala como una pedagogía importante para mejorar los procesos educativos.

Los resultados son muy interesantes, definitivamente muestran las preferencias de los profesores al momento de desarrollar su actividad académica. Hay que subrayar que la pedagogía de la Investigación-Acción es con mucha ventaja la más citada. La segunda de preferencia docente es la pedagogía Crítica. En el otro extremo ningún profesor citó la pedagogía Postestructuralista.
Desde otro punto de vista, cada modelo pedagógico implica para el profesor, no solo la labor o rol habitual de la docencia, sino, según señala Marina en la página 229 de su libro, que los profesores deben, además de esa docencia, que, como el valor en la guerra, se le supone al docente convertirse en:

1. Agentes de Cambio.

2. Facilitadores Experienciales.

3. Diseñadores de Entornos de Aprendizaje.

4. Arquitectos de la Cultura de la Clase.

5. Impulsores del Aprendizaje Profundo.

6. Guías.

7. Directores de Experiencia.

8. Entrenadores.

9. "Coaches"

10. Tutores.

11. Conectores.

12. Mentores.

13. Evaluadores.

14. Educadores Emocionales.

15. Educadores de Inteligencias Múltiples.

16. Fomentadores del Flujo. Etc. (Marina, 2017)

Evidentemente el profesor se encuentra envuelto en una paradoja, ya que todos estos potenciales roles implican una nueva visión y preparación académica, sin que, en la mayoría de 
los casos, se produzca una específica formación del docente. O sea: ¿Cómo ser profesor y todos estos roles al mismo tiempo y en el mismo establecimiento?

Parece que los profesores deben convertirse en una especie de "Hombre Orquesta" capaces de multiplicar casi hasta el infinito sus habilidades, sin que evidentemente se les ayude mediante preparación específica o se les gratifique de alguna manera, no solo lo monetario tiene importancia, para que cumplan estos nuevos roles.

De manera que planteamos una problemática ¿Para el desarrollo local qué pedagogía y qué roles deben desempeñarse a nivel de la docencia?

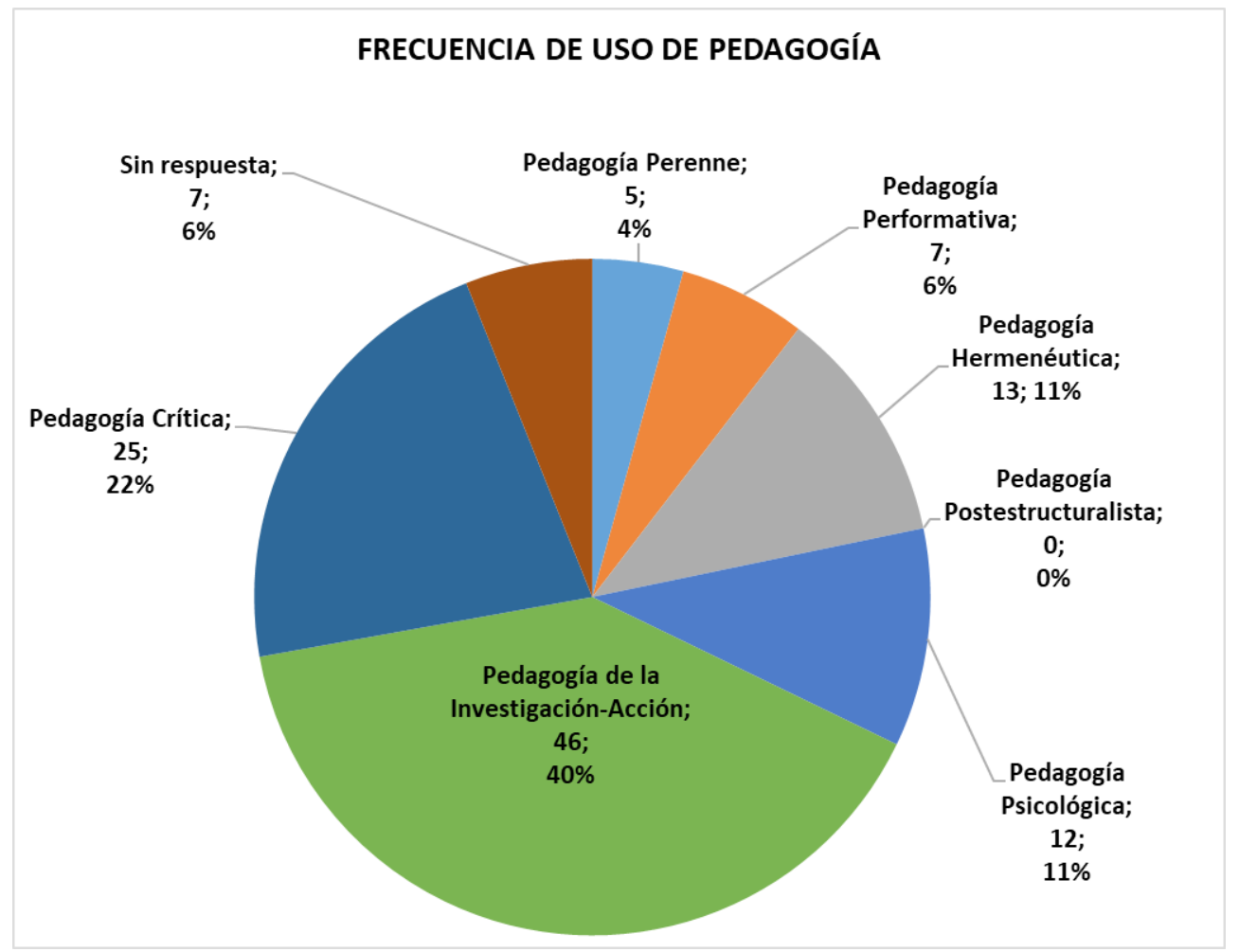

Figura 1. Pedagogía utilizada en clase.

Fuente: Asistentes a Primer Congreso Internacional de Ciencias Humanas y Educación, 2018

Elaboración: Propia 
¿Qué papel puede jugar la educación

\section{en el desarrollo local?}

Si está claro, que, en todas las aproximaciones metodológicas conocidas sobre desarrollo local, la educación es considerada como un factor sumamente importante para conseguir dicho desarrollo. Aunque en la mayoría de los casos esto no pasa de ser una afirmación sin contextualizar, a nivel de declaraciones de principios.

Para tener una idea un poco más completa del papel que puede jugar la educación en el desarrollo local, en la conferencia de Pujilí se recomendó la lectura crítica del documento citado en la bibliografía de Gabriela Orduna Allegrini. En él se hace un interesante análisis de esta temática, así en la página 72 se indica:

“...se puede afirmar que la educación es un medio, un instrumento para alcanzar el desarrollo local, empleado por el grupo social para:

- afirmar la personalidad de los individuos, enriqueciéndoles con la capacidad de afrontar las contingencias de la vida y crear oportunidades de superación,

- establecer las mejores relaciones recíprocas entre sus miembros,

- velar por la conservación de los valores del patrimonio cultural,

- promover y afianzar en los individuos la adquisición de intereses que procuren un mayor bienestar individual y colectivo,

- y orientar las múltiples actividades de la vida social hacia metas que aseguren una existencia más próspera y feliz" (Orduna Allegrini, 2003)

Y en la página 73 se responde en parte a la pregunta ¿Qué relación entre educación y desarrollo? Citando a quién es considerado uno de los padres de la escuela francesa de sociología rural y gran investigador de este tema del desarrollo, sobre todo en su faceta rural.

“Louis Malassis (1975, p.100) en su obra "Ruralidad, Educación y Desarrollo", establece claramente la relación entre ambos procesos:

"La educación es condición necesaria del desarrollo. Constituye la base fundamental del sistema de creacióndifusión que impulsa el proceso técnico, elemento fundamental del crecimiento y la productividad.

La educación es una condición necesaria, pero no suficiente del desarrollo, el cual implica la creación o extensión de las instituciones motivadoras y un instrumento de transformación social: contribuye al crecimiento en razón de la invención y su difusión y si dispone de autonomía suficiente, es también instrumento de desarrollo por el análisis crítico" (Malassis, 1975)

En el mismo trabajo de Gabriela Orduna, en la página 73 , se señala que: 
"Se puede, también, añadir la idea de que la educación es condición necesaria del desarrollo en la medida en que, como proceso de comunicación humana, establece los cauces adecuados para que los propios individuos, aprendan a integrarse en los grupos comunitarios, sean capaces de participar en la organización social de mejoras locales y estén capacitados para emprender acciones que faciliten el aprovechamiento óptimo y sostenible del resto de los recursos locales." (Orduna Allegrini, 2003)

(Pallasco, 2009) en Objetivos de la nueva

Escuela de la Educación para la Emancipación escribe:

"Como una alternativa para enfrentar la actual situación y proyectar los alcances de una nueva educación en el país, los maestros ecuatorianos hemos desarrollado una propuesta que parte y corresponde a la realidad del Ecuador, que busca y contribuye a la transformación, no solo educativa, sino a la transformación de la sociedad toda.

Planteamos establecer una Escuela que forme educandos para el trabajo y para la vida. ... Una Escuela para todos los niños y niñas, para todos los jóvenes de la ciudad y el campo, para todos los pueblos, en la que nadie se quede al margen de la educación; obligatoria, desde la educación inicial, en sus diversas modalidades, hasta el bachillerato... Una Escuela imbricada con el desarrollo social, económico, cultural y político de la comunidad, que fortalezca los nexos de sus integrantes, que se convierta en foro para el debate, en escenario para la toma de decisiones, y en eje del desarrollo comunitario... Una Escuela que revolucione los métodos de enseñanza-aprendizaje, que medie democráticamente entre los saberes y los alumnos. Una educación que afirme la Independencia y Soberanía del Ecuador en el marco de la unidad internacionalista de los trabajadores y los pueblos de la Tierra; que contribuya al progreso social y material del país, que abone para la disminución de las diferencias científicas y tecnológicas con los países desarrollados". (Pallasco, 2009)

En el artículo publicado en la página oficial de la Presidencia de la República del Ecuador bajo el título Presidente Lenín Moreno inauguró Unidad Educativa que beneficia a comunidades de Cangahua, el 14 de noviembre de 2018, en su intervención en el acto de inauguración de la Unidad Educativa GuachaláMitad del Mundo, el presidente de la República, entre otras cosas, puntualizó:

“ ¡El aprendizaje nunca será un gasto y jamás puede detenerse! Hemos dicho y repetido, y nunca nos cansaremos de hacerlo, que el camino más corto para salir de la pobreza jes la educación!”...

"Este es un ejemplo que vamos a seguir replicando en el país. Siempre debemos recordar que la educación es tarea de todos" .... Mientras, el programa Nueva Escuela refiere a un innovador estándar para la construcción de instituciones educativas sostenibles. Este modelo responde a las necesidades del territorio en el ámbito social, económico, ambiental y pedagógico. Sus lineamientos generales hacen énfasis en diseño bioclimático, sistemas constructivos y materiales de apropiados para el territorio; eficiencia energética, manejo de desechos, y aportes pedagógicos. "Se han establecido los estándares que deben cumplir -poco a 
poco- todas las instituciones educativas, para alcanzar niveles óptimos de calidad. Además de cumplir con la tarea de educar, buscamos que sean centros de participación comunitaria, que promuevan el desarrollo de cada localidad". (Presidencia de la República del Ecuador, 2018)

Esta información engrana de manera perfecta en el presente análisis que búsqueda del verdadero papel de la pedagogía en el desarrollo de los pueblos. El mensaje del presidente se encamina a dirigir toda la atención al ámbito educativo para buscar un mejor nivel de vida y de desarrollo de los pueblos.

Se podría decir resumiendo, y en tono coloquial, que la educación es condición necesaria pero no suficiente para conseguir un desarrollo local, correcto. Hay que subrayar de todas formas que evidentemente por si sola, la educación por muy importante que parezca, no es la única e imprescindible palanca, para conseguir el desarrollo local.

\section{Una propuesta}

Una vez indicados los elementos previos se podría preguntar: ¿Qué podemos hacer para contribuir con el desarrollo sostenible?

En primer lugar, intentar incorporar un paradigma concreto a nuestra enseñanza, una opción, en el sentido de que puede ser el más avanzado, hay que recalcar que es una preferencia de los autores y como tal debe ser considerada. Habría que precisar que se habla del desarrollo local sostenible, derivado del desarrollo sostenible.

La opción puede ser sometida a cualquier tipo de crítica, ya que, es conocido que el término desarrollo sostenible es extremadamente ambiguo y ha servido sistemáticamente para realizar múltiples tropelías, muchas de las cuales están en el recuerdo de todos los que conocen un poco, el debate en torno al término.

Obviamente, al menos, hay otro paradigma importante formándose en Latinoamérica; es el del Postdesarrollo pero aunque sus autores son importantes y conocidos: Walter Mignolo, Arturo Escobar, Eduardo Gudynas, desde mi punto de vista sus aportaciones no se concretan de manera operativa. Es innegable la potencia que este paradigma tiene a nivel teórico y que su crítica debe ser analizada y estudiada detalladamente, pero si hablamos de los niveles metodológico y técnico la opinión de los autores es que su validez a este nivel deja bastantes huecos difícilmente rellenables.

\footnotetext{
Por ello, dada la necesidad de realidades
} 
operativas para poder empezar a trabajar ¡Ya!, se analiza y se sugiere el trabajo realizado por un grupo de investigadores franceses quienes explican sus propuestas para trabajar en establecimientos escolares acerca del desarrollo sostenible y resumen su trabajo así:

"En el espacio de quince años, el desarrollo sostenible ha interferido con todas las estrategias nacionales y los informes resumidos de las principales instituciones internacionales (OCDE, Banco Mundial, PNUD ...). Se presenta en forma de educación para el desarrollo sostenible (UNESCO, década 2005-2014) $\mathrm{y}$ en forma de indicadores que pueden medir el progreso realizado. Los ODM, y luego los ODS, están recibiendo una atención especial en la actualidad. Los estados buscan alcanzar por todos los medios los objetivos prescritos por los 17 ODS para 2030, el PNUD tiene la intención de ayudar a los países que están luchando por mantener el rumbo y las ONG están tratando de comprender los problemas asociados con estos nuevos objetivos. Entre estos ODS, el SDG 4 La educación de calidad es de suma importancia. Más allá del hecho de que la educación es una variable clave en el desarrollo de un país, SDG 4 se posiciona como un factor clave de cambio, un cambio que es más cualitativo que cuantitativo porque asume que el desarrollo sostenible (y su) conduce a cambios reales en los comportamientos individuales". (Diemer, Arnaud \& Khushik, Faheem \& Ndiaye, Abdourakhmane., 2017)

No significa una adscripción acrítica a lo que se dice en ese documento, pero si una especie de guía de actuaciones que pueden significar el inicio de unas mejoras evidentes en el caso de aplicarse a establecimientos escolares, colaborando de esa manera al desarrollo local, sobre todo y de manera clara desde el punto de vista del medioambiente.

Así los autores indican una serie de reflexiones que se considera adecuado y razonable ponerlas en práctica:

1. "La educación para el desarrollo sostenible permite a cada uno adquirir los conocimientos, las competencias, las actitudes y los valores necesarios para construir un porvenir sostenible.

2. La educación para el desarrollo sostenible consiste en integrar en la enseñanzay elaprendizaje, los temas clave del desarrollo sostenible, como el cambio climático, la prevención de riesgos naturales, la biodiversidad, la reducción de la pobreza o el consumo sostenible. (EOI, 2016)

3. Implica la adopción de métodos participativos pedagógicos dirigidos a motivar y autonomizar a los alumnos para que modifiquen sus comportamientos y se vuelvan los actores del desarrollo sostenible. Es por ello que la educación para el desarrollo sostenible favorece la adquisición de competencias que permiten a los alumnos desarrollar su espíritu crítico, imaginar escenarios alternativos y tomar decisiones en común.

4. La educación para el desarrollo sostenible implica un cambio en profundidad de la enseñanza tal y como se practica hoy en día" 
(Diemer, Arnaud \& Khushik, Faheem

\& Ndiaye, Abdourakhmane., 2017)

En este caso el acuerdo es necesario respecto a estas reflexiones, pero parece que nadie en principio debería considerar incorrectas estas reflexiones.

En segundo lugar, un aspecto importante sería el de considerar: ¿Cuáles son las acciones de un Plan Verde en mi establecimiento?

Para ello se indican un total de nueve desafíos que a continuación se indican:

"Primer desafío: Consumo y producción sostenible. Menos papeles, menos residuos. Un plan biológico en la cantina.

Segundo desafío: Formación e investigaciones sobre el desarrollo sostenible. Conferencias, cursos, jornadas, tesis, un laboratorio sobre desarrollo sostenible.

Tercer desafío: Gobernanza, implicación de la dirección, del personal y de los estudiantes en las acciones de desarrollo sostenible del establecimiento, carta de desarrollo sostenible, informe anual, información y labialización de acciones de desarrollo sostenible.

Cuarto desafío: Cambio climático y energías. Balance carbono de los edificios y de las actividades. Acciones de eficiencia energética.

Quinto desafío: transporte y movilidad sostenible. Balance carbono de los transportes de los estudiantes y del personal. Acciones para reducir los desplazamientos. Aparcamientos para bicicletas.
Sexto desafío: Conservación y gestión sostenible de la biodiversidad y de los recursos naturales: Menos consumo de agua. Observatorio de los animales de la zona. Menos herbicida en los jardines.

Séptimo desafío: Salud pública selección y gestión de riesgos: Acciones contra las enfermedades de transmisión sexual. Drogas, tabaco, desequilibrios alimentarios, stress, acoso, etc.

Octavo desafío: Inmigración, inclusión social, Acciones para la equidad, proyectos de lucha contra la exclusión.

Noveno desafío: Desafíos internacionales en materia de desarrollo sostenible y de pobreza a nivel mundial. ¿Cuáles acciones?" (Diemer, Arnaud \& Khushik, Faheem \& Ndiaye, Abdourakhmane., 2017)El considerar estos desafíos en un establecimiento educativo puede llevar a realizar un cambio importante en su relación con el medioambiente y con el desarrollo local sostenible. Se trata de una primera aproximación que puede ser útil para empezar a cambiar de registro respecto a las actividades habituales dentro de los establecimientos educativos y de alguna manera cambiar la tendencia pesada que en la actualidad domina estos campos del conocimiento.

Finalmente indicar que:

"El territorio puede desplegar el desarrollo sostenible en sus políticas de empleo, cultura, ordenación territorial, transportes, turismo, urbanismo. etc. Dentro de esas políticas los establecimientos pueden colaborar"

\section{Conclusiones}

La educación juega un rol importante en el desarrollo de los pueblos, pero no se puede 
endosarle la responsabilidad total en lo referente al desarrollo local sostenible.

Desde los estamentos gubernamentales y sociales existe un discurso que se entiende claramente como una transferencia de responsabilidades del crecimiento económico, (o al menos de las que se relacionan directamente a las actividades económicas), hacia la actividad académica que se desarrolla en las aulas y en las instituciones educativas. El mensaje presidencial pone la mirada en el ámbito educativo para buscar un mejor nivel de vida y de desarrollo de los pueblos.

La presencia de docentes en el Primer Congreso Internacional de Ciencias Humanas y Educación permitió obtener información de primera mano acerca de las corrientes pedagógicas utilizadas por ellos en el aula para desarrollar el proceso académico y facilitó la obtención de resultados que nos llevan creer firmemente que la pedagogía de la investigaciónacción es la más apropiada para utilizarla como medio de alcanzar un desarrollo local. Hay que resaltar que la pedagogía de la InvestigaciónAcción es la más citada.

Los resultados permiten identificar el uso variado de pedagogías por parte de los docentes y se determina así que la educación es solo un instrumento para alcanzar el desarrollo local.

A través de la educación el ser humano adquiere conocimientos, desarrolla habilidades y competencias, desarrolla su espíritu crítico pone en práctica y enriquece sus valores éticos y morales, toma decisiones en común y de esta manera se prepara para construir un porvenir sostenible. Se trata entonces de integrar cada uno de estos elementos de modo que se encaminen a solucionar los problemas más agravantes de la región.

Los modelos pedagógicos enfocan el rol del docente, no solo para la labor académica, sino como facilitadores del proceso educativo. Es decir, el docente ya no solo debe ser el guía, amigo y facilitador del conocimiento ante sus estudiantes, sino, debe ser quien impulse, dirija y promueva el desarrollo local. De esta manera se pretende alcanzar la solución a los problemas sociales desde el campo de la educación mas no desde el campo de la economía.

Debe clarificarse cuál es la pedagogía que contribuya con el desarrollo local y amerita ser adoptada por las instituciones educativas como modelo pedagógico con la clara conciencia que así se proyectará al proceso educativo hacia el 
desarrollo local.

Se debe incorporar un modelo educativo concreto, que refleje la realidad nacional y que permita el desarrollo local de acuerdo a las potencialidades de cada área territorial.

El modelo pedagógico aplicado en establecimiento franceses demuestra ser una de las mejores alternativas para iniciar con un proceso que nos enrumbe a contribuir con el desarrollo local. Pero consideramos imperios clarificar cuál de los dos grandes paradigmas existentes, Desarrollo o Postdesarrollo se implementará. Una propuesta razonable de aplicación de paradigma para el desarrollo local puede ser el desarrollo local sostenible.

Debe existir una política ministerial respecto al papel de los establecimientos académicos en relación con el desarrollo local.

\section{Referencias bibliográficas}

Carbonell, J. (2016). Pedagogías del siglo XXI. Alternativas para la innovación educativa. (Cuarta ed.). Octaedro.

Coraggio, J. (2001). Educación y desarrollo local. Obtenido de https://www. coraggioeconomia.org/jlc/archivos $\% 20$ para\%20descargar/eduydesarrollolocal. pdf

Diemer, A.; Khushik, F. \& Ndiaye, A. (2017). De la Educación para el Desarrollo Sostenible (EDS) a los Objetivos de Desarrollo Sostenible (ODS), inuevos requisitos para los países del Sur? Obtenido de https://www.researchgate. net / publication/325473036 De_l'Education_au_Developpement Durable_EDD_aux_Objectifs_du Developpement_Durable_ODD_de nouvelles_prescriptions_pour_les_pays du_Sud

Díez, E. (2009). Globalización y Educación Crítica. (D. Abajo, Ed.) Obtenido de https://buleria.unileon.es/ bitstream/handle/10612/2219/ GlobalizacionyEducacionCritica. pdf? sequence $=1$

EOI. (2016). La Educación para el Desarrollo Sostenible (EDS). Obtenido de Escuela de organización industrial: http://www. eoi.es/blogs/msoston/2016/03/29/laeducacion-para-el-desarrollo-sostenibleeds/

Hernaiz, I.; Sanjinés, E. \& Villarán, V. (2005). Educación y desarrollo local: tensiones $y$ perspectivas; Reflexiones sobre experiencias en la región andina. Obtenido de https://unesdoc.unesco.org/ ark:/48223/pf0000142558

Malassis, L. (1975). Ruralidad, educación y desarrollo. (UNESCO, Ed.) Buenos Aires.

Marina, J. (2017). El Bosque Pedagógico. Barcelona: ARIEL.

Neira, T. (1999). Teorías y modelos de enseñanza: Posibilidades y límites. Milenio.

Orduna Allegrini, G. (2003). Desarrollo local, educación e identidad cultural. Obtenido de https://core.ac.uk/download/ pdf/83560436.pdf

Pallasco, M.(2009). Objetivos de la nueva Escuela de la Educación para la Emancipación. Red Voltaire. Obtenido de http://www. voltairenet.org/article160181.html

Picardo, O. (2005). Diccionario Pedagógico. 
Obtenido de http:/online.upaep.mx/ campusvirtual/ebooks/diccionario.pdf

Prats, E. (2010). (E. M. SL, Ed.) Obtenido de Teorías de la educación.: http:// openaccess.uoc.edu/webapps/ o $2 /$ bitstream/10609/51061/5/ Teor $\%$ C $3 \%$ ADas $\% 20$ de $\% 201 \mathrm{a} \% 20$ educaci $\% \mathrm{C} 3 \% \mathrm{~B} 3 \mathrm{n} \_\mathrm{M} \% \mathrm{C} 3 \% \mathrm{~B} 3 \mathrm{dul} 4$ Teor $\%$ C3\%ADas $\% 20$ de $\% 201 a \% 20$ educaci $\% \mathrm{C} 3 \% \mathrm{~B} 3 \mathrm{n}$.pdf

Prats, E. (2010). Teorías de la educación. Obtenido de http://openaccess.uoc.edu/ webapps/o2/bitstream/10609/48881/5/ Teor $\%$ C3\%ADas $\% 20$ de $\% 201$ a $\% 20$ educaci $\% \mathrm{C} 3 \% \mathrm{~B} 3 \mathrm{n} \_\mathrm{M} \% \mathrm{C} 3 \% \mathrm{~B} 3 \mathrm{dul} 4$ Teor $\%$ C $3 \%$ ADas $\% 20$ de $\% 201$ a $\% 2 \overline{0}$ educaci $\% \mathrm{C} 3 \% \mathrm{~B} 3 \mathrm{n}$.pdf

Prats, E. (2015). Teorías de la educación. Obtenido de http://openaccess.uoc.edu/ webapps/o2/bitstream/10609/51061/5/ Teor $\%$ C3\%ADas $\% 20$ de $\% 201$ a $\% 20$ educaci $\% \mathrm{C} 3 \% \mathrm{~B} 3 \mathrm{n} \mathrm{M} \% \mathrm{C} 3 \% \mathrm{~B} 3 \mathrm{dul} 4$ Teor $\%$ C $3 \%$ ADas $\% 20$ de $\% 201$ a $\% 20$ educaci $\% \mathrm{C} 3 \% \mathrm{~B} 3 \mathrm{n} . \mathrm{pdf}$

Presidencia de la República del Ecuador. (2018). Obtenido de Presidencia de la República del Ecuador: https://www. presidencia.gob.ec/presidente-leninmoreno-inauguro-unidad-educativa-quebeneficia-a-comunidades-de-cangahua/

Razeto, L. (2000). Educación popular y desarrollo local. Obtenido de http://base. socioeco.org/docs/doc-7310_es.pdf

Soler, J. (2009). La renovació pedagogica durant el segle $X X$. Obtenido de http://diposit. ub.edu/dspace/bitstream/2445/43097/1/ JSM_TESI.pdf

Stiglitz, J. \& Greenwald, B. (2016). La creación de una sociedad del aprendizaje: Una nueva aproximación al crecimiento, el desarrollo y el progreso social. La Esfera de los Libros. siglo XX para la escuela del siglo XXI. Barcelona: Graó, de IRIF, S.L. 